\title{
ISPAD Clinical Practice Consensus Guidelines 2018: Sick day management in children and adolescents with diabetes
}

\author{
Lori M. Laffel ${ }^{1} \odot \mid$ Catarina Limbert ${ }^{2} \mid$ Helen Phelan $^{3} \odot$ | Anju Virmani ${ }^{4} \mid$ Jamie Wood $^{5} \mid$
}

Sabine E. Hofer ${ }^{6}$

${ }^{1}$ Joslin Diabetes Center, Harvard Medical School, Boston, Massachusetts

${ }^{2}$ NOVA Medical School, CHLC-Hospital Dona Estefania, Lisbon, Portugal

${ }^{3}$ John Hunter Children's Hospital, Newcastle, New South Wales, Australia

${ }^{4}$ Max, Pentamed \& SL Jain Hospitals, Delhi, India

${ }^{5}$ University Hospitals Cleveland Medical Center, Case Western Reserve University, Cleveland, Ohio

${ }^{6}$ Department of Pediatrics, Medical University of Innsbruck, Innsbruck, Austria

Correspondence

Lori M. Laffel, MD, MPH, Pediatric, Adolescent \& Young Adult Section, Section on Clinical, Behavioral \& Outcomes Research, Joslin Diabetes Center, Harvard Medical School, One Joslin Place, Boston, MA 02215.

Email: lori.laffel@joslin.harvard.edu

\section{1 | WHAT'S NEW IN SICK DAY MANAGEMENT?}

- The tried and true "Back to the Future" approach of frequent monitoring of glucose and ketones, preferably of blood over urine ketones, with timely administration of supplemental insulin along with 24 hour, 7 day a week access to expert health care team advice can successfully manage sick days and prevent progression to diabetic ketoacidosis (DKA) in young persons with insulin-treated diabetes.

- Use of continuous glucose monitoring (CGM) devices may aid in sick day management; greater penetration of CGMs with use of CGM trend arrows for insulin dose adjustments can reduce glycemic excursions and provide benefit.

- There are particular clinical situations when ketogenesis can be common, such as in the setting of disordered eating behaviors, use of SGLT1/2 inhibitors, and low carbohydrate diets, when frequent glucose and ketone monitoring along with sick day management are needed to prevent development of DKA.

\section{2 | EXECUTIVE SUMMARY AND RECOMMENDATIONS}

The diabetes care team should provide clear guidance to patients and families on how to manage diabetes during intercurrent illnesses, and how to contact the diabetes team as well as emergency medical personnel, if needed (24-hour diabetes team telephone contacts, mobile telephones, emergency medical assistance procedures, etc.). In addition to intercurrent illnesses, patients and families should feel comfortable and be encouraged to call for help at times of mismanagement, either accidental (missed insulin doses, incorrect timing of doses [eg, AM given in PM], pump occlusion, etc.) or intentional (insulin restriction, etc.) in order to avoid prolonged out-of-range glucose levels and progression either to diabetic ketoacidosis (DKA) or severe hypoglycemia. Reeducation should be repeated annually, for example, at the time of annual flu shot administration, in order to avoid:

- uncontrolled or symptomatic hyperglycemia

- dehydration

- ketoacidosis

- severe hypoglycemia

Patients and families/care providers should be reminded:

- Never to stop insulin completely [A]

- When vomiting occurs in a child or adolescent with diabetes, it should always be considered a sign of insulin deficiency until proven otherwise $[\mathrm{E}]$

- The insulin dose usually needs to be increased (in addition to usual dose) when there is fever, with many acute illnesses (except for GI illnesses with emesis), during/following surgical procedures, treatment with corticosteroids (eg, asthma flare, poison ivy, nephrotic syndrome, etc.), and with any major stress based on knowledge of clinical symptoms and signs and, especially, with 
awareness of monitored blood glucose and blood (or urine) ketone levels checked frequently [E]:

1. Elevated blood glucose with absence or only small amount of ketones:

- Give additional $5 \%$ to $10 \%$ of the total daily dose (TDD) of insulin ( 0.05-0.1 U/kg) as short or rapid-acting insulin subcutaneously and repeat this same dose every 2 to 4 hours according to blood glucose response and clinical condition. Rarely, intramuscular insulin can be given although absorption may be no faster than with rapid acting analog. TDD of insulin is the sum of all long, intermediate, and short/rapidacting insulins usually taken (for insulin pump users, TDD is the sum of basal and bolus doses). One can also give $110 \%$ of the calculated correction dose, repeated every 2 to 4 hours based on blood glucose response. For insulin pump users, one can also increase the basal rate using a temporary basal rate with an increase of $\sim 10 \%$ to $30 \%$ for 2 to 4 hours or longer as needed.

2. Elevated blood glucose with moderate or large amount of ketones reflects actual or impending DKA with potential for coma or death:

- Give additional $10 \%$ to $20 \%$ of the TDD of insulin $(\sim 0.1-0.2 \mathrm{U} / \mathrm{kg})$ as short or rapid-acting insulin subcutaneously and repeat this same dose every 2 to 4 hours according to blood glucose response and clinical condition. Rarely, intramuscular insulin can be given although absorption may be no faster than with rapid acting analog. One can also give $120 \%$ of the calculated correction dose, repeated every 2 to 4 hours based on blood glucose response. For insulin pump users, one can also increase the basal rate using a temporary basal rate with an increase of $\sim 20 \%$ to $50 \%$ for 2 to 4 hours or longer as needed.

- Insulin doses may need to be increased considerably above the $10 \%$ to $20 \%$ increments in children who are in the partial remission or "honeymoon" phase when doses are relatively small; consider providing supplemental doses per $\mathrm{kg}$ as noted above $(\sim 0.05-0.1 \mathrm{U} / \mathrm{kg}$ or $\sim 0.1-0.2 \mathrm{U} / \mathrm{kg})[\mathrm{E}]$.

- Monitoring blood ketones is preferred over urine ketones when available (and affordable), and the use during illness can reduce emergency room visits and hospitalizations [B]. Where blood ketone testing is not possible, patients should be encouraged to use urine ketone strips during illnesses and when blood glucose is persistently elevated. During the recovery phase, urine ketones (which measure acetoacetate) show improvement only after a certain lag period.

- Aim for a blood glucose between 4 and 10 mmol/L (70-180 mg/dL) and blood ketones below $0.6 \mathrm{mmol} / \mathrm{L}$ when the child is ill [E].

- Use of continuous glucose monitoring (CGM) devices or intermittently scanned glucose monitoring devices is helpful as a supplement to blood glucose monitoring during illness and at times of out-of-range glucose levels but one must ensure proper use of such devices according to the manufacturer (and as emerging data become available) due to need for calibrations (for certain devices) and potential for inaccurate readings at extremes of glucose levels with certain devices and from interfering substances like acetaminophen, aspirin, vitamin C, etc. [E].

- The insulin dose often needs to be decreased when there is gastroenteritis due to limited oral intake and/or malabsorption to prevent hypoglycemia; however, one must ensure adequate basal insulin delivery to prevent hyperglycemia and hyperketonemia due to insulin deficiency $[E]$.

- Hypoglycemia with hyperketonemia, which may occur in the setting of Gl illness or starvation, requires administration of insulin along with carbohydrate intake [E].

- Maintaining hydration is essential in every child with diabetes during an acute illness; oral fluids with or without sugar should be consumed depending on the glucose level; consider timely initiation of IV fluids if the child is unable to drink [E].

- In a child or adolescent with an intercurrent illness, URGENT specialist advice with possible referral to emergency care must be obtained when [E for all below]:

- the underlying condition is unclear, fever persists, or family members are uncomfortable providing home care for any reason

- weight loss continues, suggesting worsening dehydration and potential circulatory compromise

- vomiting persists beyond 2 hours (particularly in young children)

- parents are unable to keep blood glucose above $3.9 \mathrm{mmol} / \mathrm{L}$ (70 mg/dL)

- blood glucose level continues to rise despite extra insulin doses

- fruity breath odor (acetone) persists or worsens

- blood ketones remain elevated $>1.5 \mathrm{mmol} / \mathrm{L}$ or urine ketones remain large despite extra insulin and hydration

- the child or adolescent is becoming exhausted, confused, hyperventilating (Kussmaul breathing), or has severe abdominal pain

- there is a change in neurologic status, mental confusion, loss of consciousness, seizures, or progression of confusion to avoid potential for cerebral edema; treatment of cerebral edema is a medical emergency requiring immediate assistance with advanced medical facilities to prevent morbidity and mortality

- the child is very young ( $<5$ years old)

- there are comorbid conditions besides diabetes, making sick day management more complex (eg, Down Syndrome, disordered eating behaviors, mental illness, epilepsy, inflammatory bowel disease, malaria, parasitic infections, etc.)

- patients/relatives are exhausted or do not have the facilities or capability to provide needed care (eg, intellectual, emotional, and/or financial constraints; unavailability of insulin or other monitoring supplies, etc.)

- caretaker understanding/language problems make it difficult to communicate with the family

- the patient and/or adult caretakers request advice and/or emergency medical consultation; appropriate transport should be facilitated as soon as possible according to local circumstances; the diabetes team should assist by contacting local 
medical personnel, if possible, to ensure systems in place for initial glucose and electrolyte monitoring along with IV fluids and insulin, if needed, for emergency treatment while awaiting evacuation to higher level facilities, as indicated.

An episode of DKA in a child with established diabetes could be a signal that diabetes education along with assessment of family dynamics needs thorough review, including home blood glucose and ketone monitoring, insulin handling (injection, transport, storage, dosage, ability to adjust doses, etc.), potential for insulin manipulation, nonadherence, family problems (or even abuse), as well as routine sick day management. In addition, it may be useful to explain to family members of low socioeconomic levels and those paying out of pocket for insulin and supplies, who may restrict dosing as a means to save resources, that even a single hospital admission is more expensive than several weeks or months of home BG monitoring with insulin administration, and, further that any episode of DKA could be life threatening (see also Diabetic ketoacidosis and hyperglycemic hyperosmolar state: A consensus statement from the International Society for Pediatric and Adolescent Diabetes).

\section{3 | THE EFFECTS OF ILLNESS ON DIABETES}

Children and teenagers whose diabetes is under good metabolic control should not experience more illness or infections than children without diabetes. However, even routine childhood illnesses can make diabetes management more challenging and increase the risk for DKA or hypoglycemia (with gastroenteritis). While there are very few well controlled, prospective studies about intercurrent illness in type 1 diabetes, one study of adult patients with type 1 diabetes reported a higher risk of urinary tract, bacterial skin, or mucous-membrane infections although upper respiratory-tract infections were no more frequent in patients with type 1 diabetes than in controls. ${ }^{1}$ There is some evidence of impaired leukocyte function in poorly controlled diabetes and children with poor metabolic control may have altered immune function, increasing susceptibility to and delayed recovery from infection. $^{2}$ One pediatric study found low IgG concentrations and reduction in complement protein 4 , variant $B$ (C4B) levels related to impaired metabolic control. ${ }^{3}$ It is tempting to believe - but not scientifically validated - that chronic hyperglycemia might be associated with more problems. ${ }^{4}$ In many parts of the world, pediatric and adolescent diabetes care is woefully inadequate because of a general lack of resources, lack of health care systems, lack of expert diabetes care, as well as enormous costs of diabetes care. These factors may contribute to chronic under-insulinization or marked glycemic variability in youth, especially when insulin is too expensive, unavailable, or when there is profound fear of hypoglycemia with inability to monitor glucose levels adequately due to cost. In developing as well as developed countries, occurrence of DKA remains a problem, therefore one must have a high index of suspicion, especially among youth with poor metabolic control, that risk for DKA is high ${ }^{5}$ and prevention is key with appropriate monitoring, insulin administration, and management of intercurrent illnesses. ${ }^{4,6,7}$ In fact, a recent publication from national surveillance DKA in the United States reported that hospitalization rates for DKA have risen more than 50\% from 2009 to 2014 for persons $<45$ years of age. ${ }^{8}$ Infections and DKA continue to account for significant mortality: a study from north India showed that infections and DKA were the commonest causes of death in type 1 diabetes: $51.2 \%$ and $41.5 \%$, respectively. ${ }^{9}$

Some illnesses, especially those associated with fever, raise blood glucose levels because of higher levels of stress hormones promoting glycogenolysis, gluconeogenesis, and insulin resistance. ${ }^{10}$ Illness often increases ketone body production due to inadequate insulin levels and the counter-regulatory hormone response. In contrast, illness associated with vomiting and diarrhea (eg, viral gastroenteritis) may lower glucose levels with the increased possibility of hypoglycemia rather than hyperglycemia. Decreased food intake, poor absorption, delayed gastric emptying, and/or overt diarrhea with more rapid transit time during gastroenteritis may contribute to hypoglycemia risk. Sometimes, one may see increasing insulin requirements during the incubation period of an infection for a few days before the onset of the illness. The increased need for insulin may persist for a few days after the illness has passed, presumably due to insulin resistance, but insulin needs are highly variable from one person to another and from one illness to the next. In the midst of a typical viral "epidemic," however, patterns may occur that facilitate making some generalizations to help advise subsequent patients/families.

\section{Five general sick day diabetes management principles:}

1. Sick day guidelines, including insulin adjustments, should be taught soon after diagnosis and reviewed at least annually with patients and family members in order to reduce risk for DKA and for severe hypoglycemia (with Gl illnesses).

2. More frequent blood glucose and ketone (blood or urine) monitoring.

3. DO NOT STOP INSULIN.

4. Monitor and maintain hydration with adequate salt and water balance.

5. Treat any underlying, precipitating illness.

\section{1 | Sick day guidelines, including insulin adjustments, should be taught soon after diagnosis and reviewed at least annually with patients and family members in order to reduce risk for DKA and for severe hypoglycemia (with GI illnesses)}

\section{Sick day preparation}

All families should receive education on sick day management. At diagnosis, families can be overwhelmed with new information and it is common for them to not retain information about sick day management postdiagnosis. ${ }^{11}$ For this reason the information at diagnosis should be simple, focusing on the importance of frequent monitoring and not stopping insulin during an illness, and calling the health care 
team early for advice. As families become more competent with their diabetes care, the sick day management education should be repeated and extended to include specific education about the effects of intercurrent illness, hyperglycemia, ketosis, symptoms of DKA, DKA prevention, sick day diet and fluids, and management of hypoglycemia with mini-dose glucagon (if approach is available locally). ${ }^{12}$

Households should maintain readily available supplies and information for sick day management including:

- Written information on management and important contact numbers/addresses of the health care team. Health care team availability by telephone has been shown in several clinical studies to facilitate communication, allow for earlier advice and institution of sick day guidelines, and decrease or minimize clinical decompensation and even avoid emergency room use as well as hospitalization. ${ }^{13-15}$

- Consider use of mobile apps for sick day management that are available on smart phones and can be used by patients/families; however, these apps should not replace communication with the health care team.

- Sick day foods and hydration supplies such as chicken soup, electrolyte drinks, etc.

- Sufficient glucose and ketone monitoring supplies, additional insulin, and an emergency glucagon kit.

The health care team should tailor the education to suit the age of the child/adolescent and development stage. ${ }^{16}$ For very young children, families should receive appropriate advice on managing gastroenteritis and the need for early intervention and possible mini-dose glucagon (see Table 2). ${ }^{12}$ Older teens should receive sick day management education as they become more independent in their diabetes self-management, although families should remain involved regardless of age, as managing any intercurrent illness can be challenging without support and guidance.

During the influenza season, health care professionals should assess families' sick day management knowledge and review sick day management plans. ${ }^{17}$ Families should be advised of the local recommendations regarding influenza vaccination. Where influenza and pneumococcal immunizations are available and recommended, for example, in the United States by the American Diabetes Association and the American Academy of Pediatrics, health care professionals should encourage families to immunize their children and address any expressed barriers to the uptake of immunizations, including concerns they may have regarding managing minor side effects.

\section{2 | More frequent monitoring}

\subsection{1 | Glucose}

- Frequent blood glucose monitoring facilitates optimal management during illness (with adult supervision, especially in adolescents). This is not difficult for children routinely using CGM; the parents and adolescent should keep a close eye on glucose trends.

- Blood glucose should be monitored at least every 3 to 4 hours, including through the night, and often every 1 to 2 hours.
- Urine glucose can be utilized if blood glucose monitoring equipment is not available., ${ }^{4,6}$

- If routine blood glucose monitoring is not available, ideally emergency glucose monitoring supplies should be "saved" for episodes of illness (making sure the strips have been stored properly and have not long expired), according to individual local circumstances. ${ }^{18}$

- Distinguishing illnesses associated with hyperglycemia from those associated with hypoglycemia is facilitated by BG monitoring. ${ }^{4,6,19,20}$

- Insulin adjustments (sick day extra doses) and other insulin changes take place in direct relationship to the ongoing blood glucose monitoring results (see below).

\subsection{2 | Ketones}

Ketones are produced by the liver from free fatty acids that are mobilized as an alternative energy source when there is lack of glucose for intracellular metabolism, either from inadequate intake or inability to utilize glucose in the setting of insulin deficiency. Starvation ketones are produced when the blood glucose is low. Ketones are also produced when insulin is lacking to initiate the transport of glucose from the blood stream into the cell. Ketones accumulate because of increased lipolysis and increased ketogenesis, due to low insulin levels and elevated counter-regulatory hormone levels. There are three ketones: acetoacetate, acetone, and beta-hydroxybutyrate. Urine ketone strips measure acetoacetate (AcAc) and acetone (if the strip contains glycine), blood ketone strips measure beta-hydroxybutyrate (BOHB), the predominant ketone in DKA, while most labs measure serum acetoacetate. In acute ketoacidosis, the ketone body ratio (BOHB:AcAc) rises from the usual level of $1: 1$ to $10: 1$ or more. ${ }^{21}$ In response to insulin therapy, $\mathrm{BOHB}$ levels commonly decrease long before AcAc levels do. The frequently employed nitroprusside test only detects AcAc in blood and urine and so routine urine ketone monitoring often shows prolonged ketonuria even when significant ketoacidosis and hyperketonemia have already responded to treatment. ${ }^{21}$ The prolonged ketonuria can result in confusion when there is clinical improvement following successful sick day management. In fact, if the persistent ketonuria leads to additional insulin administration when it is no longer needed, either at home or in emergency rooms/hospital settings due to a desire to "clear" the urine ketones, hypoglycemia can ensure. Monitoring blood ketones prevents this problem as BOHB declines directly in response to insulin therapy. In fact, declines in BOHB levels will be clinically evident even before declines in blood glucose levels. ${ }^{4,6,14,22}$ Thus, blood ketone tests (for $\mathrm{BOHB}$ ), or urine ketone tests when blood ketone monitoring is unavailable, help to guide sick day management, with blood ketone testing for BOHB providing additional information to urine ketone testing:

- Blood $\mathrm{BOHB} \geq 0.6 \mathrm{mmol} / \mathrm{L}$ is abnormal in children with diabetes. ${ }^{23,24}$

- Adult studies have shown that the time delay after a pump stop to diagnose ketosis is significantly longer for ketonuria than for plasma ketonemia ${ }^{25}$ and that urinary ketone tests can remain 
positive more than 24 hours after resolution of ketoacidosis in the majority of patients. ${ }^{26}$

- There can be a dissociation between urine ketone (AcAc) and blood BOHB concentrations such that urine ketone tests can still be negative or show only trace or small ketone levels when blood BOHB is already high, indicating need for treatment. ${ }^{4,27}$

- Following resolution of DKA, the dissociation between urine ketones and blood ketones continues as urine ketone levels remain elevated, and can lead to excess insulin administration and risk for hypoglycemia if treatment is based on the urine ketone result rather than the blood ketone result.

- Urine ketone supplies are inexpensive but may deteriorate within a month or so after opening the bottle, so care may be needed to ensure a fresh bottle is available if the previous bottle had been opened more than a few months ago.

Home measurement of blood BOHB concentrations in children and adolescents enables earlier identification and treatment of ketosis compared to urine ketone testing, and decreases diabetes-related hospital visits (both emergency department visits and hospitalizations). ${ }^{28-30}$ Patients should be encouraged to have home blood ketone test strips. However, blood ketone strips can be unaffordable for many households, may not be covered by insurance programs, or may not be available. In these circumstances, urine ketone strips can be used for sick day management. In countries where diabetes is uncommon, or a low priority, patients/families should be encouraged to carry the blood ketone strips with meter or urine ketone strips to the hospital if the child needs admission, in case the hospital does not have the facilities for ketone testing.

Blood $\mathrm{BOHB}$ measurements may be especially valuable to prevent DKA in patients who use an insulin pump, as only rapid- or shortacting insulin is used in this type of therapy. Elevations in blood BOHB may precede elevations in urine ketones due to interrupted insulin delivery $^{22}$ (eg, catheter occlusion or dislodgement), which can rapidly lead to ketogenesis and hyperketonemia, as well as increased insulin needs. During resolution of ketosis, blood BOHB normalizes sooner than urine ketones. ${ }^{4,6}$ Monitoring $\mathrm{BOHB}$ may also have the potential to help prevent late hypoglycemia from overtreatment with insulin, based upon the persistence of ketonuria while ketonemia is improving. Blood BOHB monitoring can be especially useful in very young children, who cannot provide urine on demand, or in others who find giving urine samples difficult.

\subsection{Never stop insulin}

The insulin dose may need to be increased or decreased to maintain glucose metabolism but it should never be stopped (see Table 1 for insulin dosing recommendations).

- The most common mistake made by health care providers and caregivers who are unfamiliar with diabetes is to recommend the complete omission of insulin because "the child is ill and not eating," thus increasing the risk of frank DKA., 4,6,10,18

- Even in the fasting state, some insulin is required for basal metabolic needs, which may go up during an acute illness, when counter-regulatory stress hormones are elevated, so that more frequent monitoring of blood glucose and ketones is required to direct insulin dosing.

- If episodes of hyperglycemia, ketosis, and vomiting recur, with or without infection, it should be recognized that this may be due to insulin restriction or omission. ${ }^{31}$ Insulin omission is particularly problematic during adolescence ${ }^{32}$ and often represents a severe psychosocial issue, for example, sexual or physical trauma, emotional trauma or abuse, poorly treated or unrecognized anxiety or depression, learning problems, executive dysfunction and/or attention deficit disorders, or some combination. Insulin restriction or omission also may be used as a form of purging to lose weight, representing a unique and dangerous form of disordered eating in persons with type 1 diabetes. ${ }^{33}$ Family dysfunction or a psychiatric illness in a caregiver is frequently seen in such circumstances, and may contribute to the recurrence of DKA episodes either directly or indirectly. ${ }^{34}$ Lack of appropriate adult supervision needs to be considered, with appropriate therapeutic interventions put into place, since recurrent DKA has a high association with DKA-related complications, including coma and death. ${ }^{4,6,10,18-21}$

The insulin "cold chain" should also be reviewed. If the cold chain is not maintained to the point of purchase (eg, the pharmacy may store in a refrigerator, but it may have been exposed to high temperatures earlier, at the warehouse level, for example), or if transport and storage are not optimal (eg, carrying insulin home after purchase, or packing insulin in baggage hold during a flight-insulin will freeze and then thaw), then insulin potency may be affected, leading to impaired insulin action. ${ }^{35}$ It may also be useful to inquire tactfully about number of insulin vials consumed. If the insulin is purchased out of pocket, the parents may be under-dosing due to financial constraints, but not documenting the actual doses, out of embarrassment. Insulin injection technique should be reviewed. Injecting persistently into hypertrophic areas, or not noticing the presence of a large bubble in the insulin cartridge, can lead to persistently high blood sugars, which may progress to DKA.

\subsection{Monitor and maintain hydration with salt and water}

- Hyperglycemia, fever, excessive glycosuria, and ketonuria all contribute to increased fluid losses.

- Sick day cabinets should contain supplies as above to prevent dehydration.

- Liquids for hydration should contain salt and water and not just plain water, especially if there are ongoing losses associated with vomiting or diarrhea. Chicken soup, consommé, home-made lemonade with both salt and sugar, or clear broths, are excellent sources of water and electrolytes, which may be needed to maintain hydration and to avoid water and electrolyte imbalance in conditions leading up to DKA.4,6,10,18-21 If appetite is decreased or the glucose level is falling below $10 \mathrm{mmol} / \mathrm{L}(180 \mathrm{mg} / \mathrm{dL})$, sugar-containing fluids should be considered to decrease risk of starvation ketosis, along with insulin at an adjusted dose. It may be helpful to remove excessive carbonation (bubbles) in some soft drinks to minimize potential for indigestion. This can be achieved 
TABLE 1 How to calculate sick day's insulin adjustments, hydration, and monitoring [E]

\begin{tabular}{|c|c|c|c|c|}
\hline \multicolumn{5}{|c|}{$\begin{array}{l}\text { A. Additional insulin } \\
\text { Hyperglycemia }\end{array}$} \\
\hline \multicolumn{2}{|c|}{ Ketones } & \multicolumn{3}{|c|}{ Blood glucose } \\
\hline Blood & Urine & $\begin{array}{l}>10-14 \mathrm{mmol} / \mathrm{L} \\
>180-250 \mathrm{mg} / \mathrm{dL}\end{array}$ & $\begin{array}{l}>14-22 \mathrm{mmol} / \mathrm{L} \\
>250-400 \mathrm{mg} / \mathrm{dL}\end{array}$ & $\begin{array}{l}>22 \mathrm{mmol} / \mathrm{L} \\
>400 \mathrm{mg} / \mathrm{dL}\end{array}$ \\
\hline$<0.6 \mathrm{mmol} / \mathrm{L}$ & Negative/trace & - Give ordinary bolus & $\begin{array}{l}\text { - Add }+5 \% \text { TDD or } 0.05 \mathrm{U} / \mathrm{kg} \text { to } \\
\text { ordinary bolus }\end{array}$ & $\begin{array}{l}\text { - Add }+10 \% \text { TDD or } 0.1 \mathrm{U} / \mathrm{kg} \text { to } \\
\text { ordinary bolus } \\
\text { - Oral sugar-free fluids }\end{array}$ \\
\hline $0.6-0.9 \mathrm{mmol} / \mathrm{L}$ & Trace/small & $\begin{array}{l}\text { - Add }+5 \% \text { TDD or }+0.05 \mathrm{U} / \mathrm{kg} \\
\text { to ordinary bolus or give } 105 \% \\
\text { of calculated correction bolus } \\
\text { - Oral sugar fluids }\end{array}$ & $\begin{array}{l}\text { - Add }+5 \%-10 \% \text { TDD } \\
\text { or } 0.05-0.1 \mathrm{U} / \mathrm{kg} \text { to ordinary } \\
\text { bolus or give } 105 \%-110 \% \text { of } \\
\text { calculated correction bolus } \\
\text { - Oral sugar-free fluids }\end{array}$ & $\begin{array}{l}\text { - Add }+10 \% \text { TDD or } 0.1 \mathrm{U} / \mathrm{kg} \text { to } \\
\text { ordinary bolus } \\
\text { - Oral sugar-free fluids }\end{array}$ \\
\hline $1-1.4 \mathrm{mmol} / \mathrm{L}$ & Small/moderate & $\begin{array}{l}\text { - Add }+5 \%-10 \% \text { TDD or 0.05-0.1 } \\
\mathrm{U} / \mathrm{kg} \text { to ordinary bolus or give } \\
105 \%-110 \% \text { of calculated } \\
\text { correction bolus } \\
\text { - Oral sugar fluids } \\
\text { - Extra } \mathrm{CHO}^{\mathrm{a}}\end{array}$ & $\begin{array}{l}\text { - Add }+10 \% \text { TDD or } 0.1 \mathrm{U} / \mathrm{kg} \text { to } \\
\text { ordinary bolus or give } 110 \% \text { of } \\
\text { calculated correction bolus } \\
\text { - Oral sugar-free fluids }\end{array}$ & $\begin{array}{l}\text { - Add }+10 \% \text { TDD or } 0.1 \mathrm{U} / \mathrm{kg} \text { to } \\
\text { ordinary bolus } \\
\text { - Oral sugar-free fluids }\end{array}$ \\
\hline $1.5-2.9 \mathrm{mmol} / \mathrm{L}$ & Moderate/large & $\begin{array}{l}\text { - Add }+5 \%-10 \% \text { TDD or } 0.05-0.1 \\
\mathrm{U} / \mathrm{kg} \text { to ordinary bolus or give } \\
105 \%-110 \% \text { of calculated } \\
\text { correction bolus } \\
\text { - Oral sugar fluids } \\
\text { - Extra } \mathrm{CHO}^{\mathrm{a}}\end{array}$ & $\begin{array}{l}\text { - Add }+20 \% \text { TDD or } 0.1-0.2 \mathrm{U} / \mathrm{kg} \\
\text { to ordinary bolus or give } 120 \% \\
\text { of calculated correction bolus } \\
\text { - Oral sugar-free fluids }\end{array}$ & $\begin{array}{l}\text { - Add }+20 \% \text { TDD or } 0.1 \mathrm{U} / \mathrm{kg} \\
\text { to ordinary bolus } \\
\text { - Oral sugar-free fluids } \\
\text { - If vomiting, consider IV saline } \\
+5 \% \text { glucose solution }\end{array}$ \\
\hline$\geq 3 \mathrm{mmol} / \mathrm{L}$ & Large & $\begin{array}{l}\text { - Add }+10 \% \text { TDD or } 0.1 \mathrm{U} / \mathrm{kg} \text { to } \\
\text { ordinary bolus or give } 110 \% \text { of } \\
\text { calculated correction bolus } \\
\text { - Oral sugar fluids } \\
\text { - Extra } \mathrm{CHO}^{\mathrm{a}} \\
\text { Risk of ketoacidosis! Consider DKA }\end{array}$ & $\begin{array}{l}\text { - Add }+20 \% \text { TDD or } 0.1-0.2 \mathrm{U} / \mathrm{kg} \\
\text { to ordinary bolus or give } 120 \% \\
\text { of calculated correction bolus } \\
\text { - Oral sugar-free fluids } \\
\text { rotocol and transfer to emergency de }\end{array}$ & $\begin{array}{l}\text { - Add }+20 \% \text { TDD or } 0.1 \mathrm{U} / \mathrm{kg} \\
\text { to ordinary bolus } \\
\text { - Oral sugar-free fluids } \\
\text { - If vomiting, consider IV saline } \\
+5 \% \text { glucose solution } \\
\text { partment }\end{array}$ \\
\hline
\end{tabular}

Check BG and ketones every 2 hours, repeat additional insulin if needed every 2-4 hours

\section{B. Insulin reduction \\ Normoglycemia/hypoglycemia}

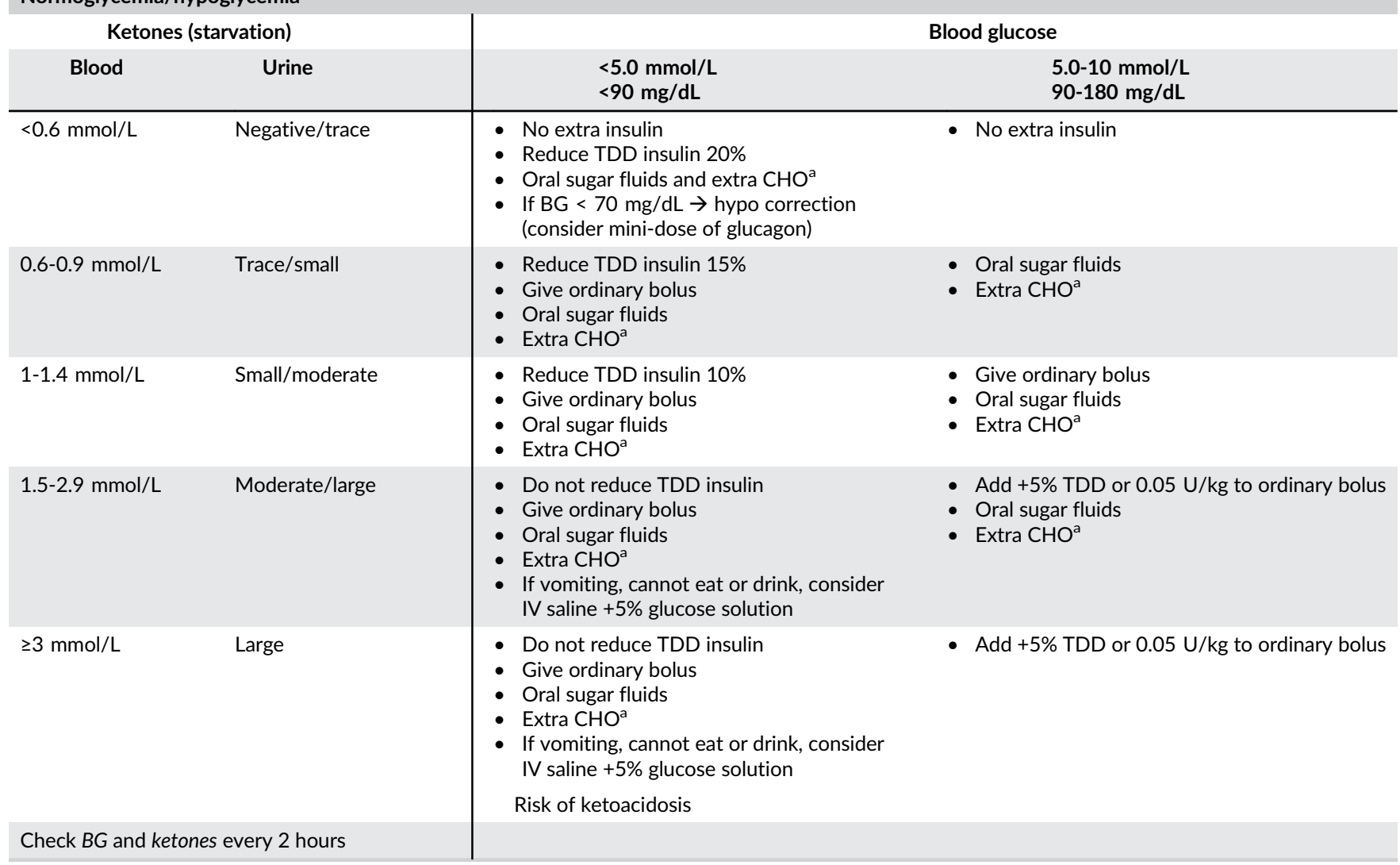




\section{TABLE 1 (continued)}

Abbreviations: BG, blood glucose; $\mathrm{CHO}$, carbohydrate; TDD, total daily dose.

Ordinary bolus = insulin dose for usual correction and/or carb intake. To calculate the TDD, add up all the insulin given on a usual day (ie, short/rapid and long/intermediate acting) or sum daily basal rates and boluses in a pump. Do not include additional boluses given for correction of hyperglycemia. In children and adolescents with usual low $(<0.7 \mathrm{U} / \mathrm{kg} / \mathrm{d})$ or usual high $(>1 \mathrm{U} / \mathrm{kg} / \mathrm{d})$ insulin requirements, consider using the percentage (\%) calculation rather than empirical 0.05 to 0.1 to $0.2 \mathrm{U} / \mathrm{kg}$ supplemental dose. High BG and elevated ketones indicate a lack of insulin. "Starvation" blood ketone levels are usually $<3.0 \mathrm{mmol} / \mathrm{L}$. When the child is feeling sick or vomiting and ketone levels are negative or low (trace or small) with BG $<10-14 \mathrm{mmol} / \mathrm{L}(<180-250 \mathrm{mg} /$ $\mathrm{dL}$ ), he/she must try to drink sugar-containing fluids in small amounts (at least $100 \mathrm{~mL} / \mathrm{h}$ ) to keep BG up. When ketone levels are elevated, priority is to give extra insulin. If BG is simultaneously low, IV saline $5 \%$ dextrose solution may be required. Additional doses of insulin are always short or rapid-acting. Short-acting insulin can be given intramuscularly to speed up absorption. The ketone level may increase slightly (10\%-20\%) within the first hour after giving extra insulin, but afterwards it should decrease. Blood ketones (BHOB) normalize sooner than urine ketones. If the child's glucose levels are persistently elevated or the illness is expected to last $\geq 3$ days, consider increasing long/ intermediate acting insulin or the basal rates delivered by pump by $10 \%-20 \%$ (even higher, up to $50 \%$ by pump at times, if needed) during the expected sick days and reduce gradually as the illness subsides [E].

${ }^{a}$ Extra carbohydrates if tolerated.

TABLE 2 Recommended dose for mini-dose glucagon

\begin{tabular}{lllll} 
& \multicolumn{2}{l}{ Quantity } & & \\
\cline { 2 - 5 } Age $(\mathbf{y})$ & Micrograms & $\mathrm{mg}$ & cc's $(1 \mathrm{mg} / \mathrm{cc})$ & $\begin{array}{l}\text { Units on insulin } \\
\text { syringe }\end{array}$ \\
$<2$ & 20 & 0.02 & 0.02 & 2 \\
$2-15$ & $\begin{array}{c}10 \text { per year } \\
\text { of age }\end{array}$ & $\begin{array}{c}0.01 \text { per year } \\
\text { of age }\end{array}$ & $\begin{array}{c}0.01 \text { per year } \\
\text { of age }\end{array}$ & $\begin{array}{c}1 \text { per year of } \\
\text { age }\end{array}$ \\
$>15$ & 150 & 0.15 & 0.15 & 15
\end{tabular}

Note that the doses recommended above are quite different (lower) from emergency doses given in case of severe hypoglycemia.

by opening the containers and allowing time for bubbles to escape along with a little shaking or stirring. Carbonated fluids may alter the distribution of food within the stomach and may contribute to bloating in some patients. ${ }^{36}$

- Elevated levels of ketones, whether associated with hypoglycemia (starvation ketosis) or hyperglycemia and insulin deficiency, can contribute to nausea and may lead to decreased food and fluid intake, exacerbating dehydration and ketosis, increasing the risk for DKA. ${ }^{4,6,10,18-21}$

- Intravenous fluids may be required if nausea, vomiting, or diarrhea are persistent with ongoing weight loss, especially in young children who are at great risk for dehydration without adequate enteral intake.

\section{Loss of appetite}

Replacing meals with easily digestible food (eg, rice-lentil broths and sugar-containing fluids) that provide energy (carbohydrates) can help prevent starvation ketosis as long as insulin is given. Necessary sick day management supplies at home include the following:

- glucose tablets, sweets, or candies such as jelly beans or sucking candies as well as dried fruits to prevent hypoglycemia

- clean (boiled/purified as necessary) water to provide hydration

- sugar and electrolyte containing fluids such as sports drinks, home-made lemonade with sugar and salt, electrolyte mixtures, or sugar-containing soft drinks or sodas to provide hydration, glucose, and salts

- easy to digest carbohydrates such as crackers, noodles, rice, or yogurt.

\section{5 | Treat any underlying precipitating illness}

\section{Specific medical advice: Treat any underlying precipitating illness}

The underlying illness should be treated as recommended for any child or adolescent without diabetes (ie, antibiotics for bacterial infections, etc.). In some parts of the world, specific endemic or epidemic illnesses have to be considered (eg, dengue hemorrhagic fever [DHF], malaria, gastrointestinal parasitic infections, etc.). Monitoring for clinical manifestations of some diseases may be complicated in patients with diabetes in whom insulin deficiency alone can present like a gastrointestinal illness with nausea and vomiting, although one would not expect any diarrhea from insulin deficiency. Treating fever, malaise, and headache with antipyretics or pain medications such as paracetamol (ie, acetaminophen) or ibuprofen is reasonable, as long as no allergies to these medications exist. Consider instructing patients and families to include acetaminophen suppositories with their sick day supplies for times when enteral intake may be difficult, such as with gastroenteritis. Additionally, one must exercise caution when taking acetaminophen or acetaminophen-containing cold medications in persons using certain CGM devices that exhibit interference from these medications. ${ }^{37,38}$ Notably, some newer generations of CGM no longer experience acetaminophen interference. ${ }^{39,40}$

Nausea and vomiting warrant careful scrutiny as vomiting can be caused by either:

i. an illness itself (ie, gastroenteritis, unclean food or food poisoning, surgical condition, other illness, etc.)

ii. severe hypoglycemia

iii. insulin deficiency resulting in hyperglycemia and ketosis, and risk for DKA.

For vomiting in association with gastroenteritis, one can consider treatment with anti-nausea medications, if available and if there is no known allergy or other medical contraindication to such treatment. Anti-nausea medications can include injectables or rectal suppositories of antiemetics (eg, ondansetron, promethazine, etc.), as oral intake of such medications may be difficult with ongoing emesis. Some patients/ families have had success with oral antiemetics like ondansetron if given in a timely manner early in the course of the illness, or just after a bout of vomiting. Such medications would be contraindicated with any mental status changes. These medications also should be used cautiously with food poisoning, when they may be contraindicated. Additionally, one must be especially cautious if the nausea and vomiting are due to DKA, when DKA management is the treatment, and not antiemetics.

Until the vomiting settles, it may be best to take small sips of cool liquids, which are better tolerated than warm liquids. Hydration can be aided by sucking on frozen pops or frozen juice bars (either sugarfree in the setting of hyperglycemia, or sugar-containing when glucose 
is low or falling). When the blood glucose is elevated and ketosis is present, extra insulin must be administered even when there is ongoing nausea and vomiting. In fact, the vomiting may stop once extra insulin has been given, due to management of the ketosis.

Oral medicines for symptomatic relief of gastroenteritis have no proven efficacy and are therefore not usually recommended. Infectious diarrheal illnesses are best managed in their locales when the local health care teams should be aware of the proper medications, and if any are indicated. Unknown or uncertain alternative medicines should be avoided; sick day education efforts should include discussion of safe and unsafe management efforts with a review of all medications.

\section{4 | INSULIN DOSING RECOMMENDATIONS FOR SICK DAY MANAGEMENT:}

\section{1 | Additional insulin:}

- Additional doses of rapid-acting or short-acting insulin are required to reduce blood glucose, turn off ketogenesis, and prevent progression to ketoacidosis and hospital admission. ${ }^{4,6,7,21}$

- The additional doses are always rapid-acting insulin analogs or short acting insulin (synthetic or animal-origin), if only the latter is available due to cost.

- The dose and frequency of injecting or bolusing will depend on the level and duration of hyperglycemia and on the severity of ketosis. Such supplemental doses are usually given subcutaneously but may also be given intramuscularly with health care professional advice.

- The additional dose of insulin is calculated as a percentage of the total of rapid/short acting and long/intermediate acting insulins for the day (or the total of bolus and basal insulin given by a pump), defined as the TDD. Supplemental doses of insulin are always given in addition to the usual insulin dose.

- If there is hyperglycemia without hyperketonemia or no more than small ketonuria, usual recommendations are to give an additional, supplemental injection or bolus of rapid-acting or shortacting insulin calculated as $5 \%$ to $10 \%$ of the TDD, or approximately 0.05 to $0.1 \mathrm{U} / \mathrm{kg}$, if the TDD is unknown. This supplemental dose, given in addition to the usual dose for carbohydrate coverage and correction, should be repeated every 2 to 4 hours, based upon blood glucose and ketone monitoring results. One can also give $110 \%$ of the calculated correction dose, repeated every 2 to 4 hours, based on response (see Table 1A).

- If there is hyperglycemia with hyperketonemia, or moderate to large ketonuria, usual recommendations are to give an additional, supplemental injection or bolus of rapid-acting or shortacting insulin calculated as $10 \%$ to $20 \%$ of the TDD, or approximately 0.1 to $0.2 \mathrm{U} / \mathrm{kg}$, if the TDD is unknown. This supplemental dose, given in addition to the usual dose for carbohydrate coverage and correction, should be repeated every 2 to 4 hours, based upon frequent blood glucose and ketone monitoring results. One can also give $120 \%$ of the calculated correction dose, repeated every 2 to 4 hours, based on response (see Table 1A). The additional dose recommendation of 0.05 to $0.2 \mathrm{U} / \mathrm{kg}$ is a general recommendation for children and adolescents with standard insulin requirements of approximately 0.7 to $1.0 \mathrm{U} / \mathrm{kg} / \mathrm{d}$. However, for children or adolescents who have low usual daily insulin requirements, or those with insulin resistance and high daily insulin requirements, the percentage calculations may work more readily rather than the empiric $\mathrm{U} / \mathrm{kg}$ additional dose.

- When children or adolescents are in the "honeymoon" remission phase and insulin doses are relatively small, there may be a need to increase supplemental insulin doses; consider providing supplemental doses per $\mathrm{kg}$ as noted above $(\sim 0.05-0.1 \mathrm{U} / \mathrm{kg}$ or $\sim 0.1-0.2 \mathrm{U} / \mathrm{kg}$ ) and assess response, as the standard supplemental dose of $10 \%$ to $20 \%$ of the TDD may be insufficient to lower the glucose levels in a timely manner.

- During illness it may also be necessary to increase basal insulin doses, whether given as a long-acting insulin analog or intermediate insulin in injection-based therapy, or as a basal rate when using an insulin pump. With a pump, temporary basal rate increases of $20 \%$ to $50 \%$, or higher at times, may be needed until the blood glucose level improves and ketone levels return to normal (BOHB $<0.6 \mathrm{mmol} / \mathrm{L}$ or negative to small urine ketones). Note, it may be necessary to increase the maximum hourly basal rate that the pump can deliver when using temporary basal rate increases for sick day management.

Example: A sick child has a blood glucose level of 14 to $20 \mathrm{mmol} / \mathrm{L}$ (ie, $250-360 \mathrm{mg} / \mathrm{dL}$ ) with blood ketones of $1.5 \mathrm{mmol} / \mathrm{L}$ (or moderate urine ketones). The family is advised to give a supplemental insulin dose of $10 \%$ to $20 \%$ of the TDD (or $0.1-0.2 \mathrm{U} / \mathrm{kg}$ ) as rapid-acting (or short-acting) insulin every 2 to 4 hours until the blood glucose falls to $<14 \mathrm{mmol} / \mathrm{L}(<250 \mathrm{mg} / \mathrm{dL})$ and the blood ketones are $<0.6 \mathrm{mmol} / \mathrm{L}$ (or urine ketones are negative to small). Thereafter, any additional doses might be $5 \%$ to $10 \%$ of the TDD (or about half of the previous supplemental dose). Blood glucose should be checked every 2 to 3 hours, and blood ketones should be checked every 1 to 2 hours, as the blood ketones will fall faster than the blood glucose. Urine ketones should be checked at each void, if blood ketone monitoring is unavailable.

- After extra insulin has been given, the blood ketone level may temporarily increase by $10 \%$ to $20 \%$ for the first hour or two but should be expected to decrease thereafter.

- Urine ketones often stay elevated for many hours because of the body's conversion of blood BOHB into AcAc, which is measured with urine testing. ${ }^{4,6,21}$

- If blood ketone level is $\geq 3 \mathrm{mmol} / \mathrm{L}$ (or the urine ketones remain large) despite extra insulin and hydration, consider referral to the emergency room for assessment and IV fluids, as the risk for DKA is high (see Table 1). 


\section{2 | Infections associated with hypoglycemia}

- Infections associated with hypoglycemia usually involve the GI tract, with viral gastroenteritis as the most common. Such illnesses are often associated with nausea and vomiting, and can occur with or without diarrhea. Hypoglycemia occurs due to reduced enteral intake with nausea and vomiting, altered gastric emptying, malabsorption, and more rapid GI transit time associated with diarrhea. Occasionally, patients and families may report unexplained hypoglycemia as a prelude to viral gastroenteritis, even prior to the first bout of emesis. Additionally, hypoglycemia may continue beyond the symptomatic stage of nausea and vomiting as malabsorption may persist a few days longer as the gut heals. During such illnesses, it is reasonable to advise replacing meals with small volumes of sugar-containing drinks for calories, provided with appropriate insulin coverage, along with fluids that contain electrolytes, as noted above. A simple diet can be reintroduced that may include rice, crackers, applesauce, bananas, tea, bread, yogurt, and potatoes, for example, depending on availability and local custom. Frequent blood glucose monitoring can guide temporary insulin dose reductions, recalling that insulin should never be totally stopped. ${ }^{4,6,18-20}$

- Include sugar-containing drinks with insulin coverage.

- Give sufficient fluids to maintain hydration, keeping records of how much the child has had to drink.

- Attend to urine output and follow body weight, if available at home, every 4 to 6 hours. Steady weight suggests adequate hydration and fluid replacement, whereas ongoing weight loss usually requires contact with the health care team to assess need for emergency room assessment or hospitalization for intravenous fluid treatment.

- Reduce total daily insulin dose by $20 \%$ to $50 \%$ during $\mathrm{Gl}$ illnesses associated with hypoglycemia, generally beginning with a $20 \%$ reduction of the basal or intermediate acting insulins and a $50 \%$ reduction of the bolus dose, which may be given after eating to ensure intake of the prepared drink and/or food. Ongoing frequent monitoring is needed because an excessive dose reduction may lead to insulin deficiency and risk for ketosis and ketoacidosis.

- Check ketones along with blood glucose as a guide to determine if starvation ketosis is occurring. Such ketones in association with hypoglycemia reflect inadequate energy supply and indicate a need for carbohydrate intake with insulin.

- If hypoglycemia persists with blood glucose levels $<3.9 \mathrm{mmol} / \mathrm{L}$ or $(<70 \mathrm{mg} / \mathrm{dL}$ ) along with nausea, vomiting, anorexia, or food refusal, a modified, smaller-than-usual dose of glucagon, if available, can be given, termed "mini-dose glucagon." Mini-dose glucagon can increase the glucose level back into a safe range as long as there are adequate glycogen stores in the liver, which can be deficient following prolonged vomiting or fasting. Nonetheless, it is safe to try mini-dose glucagon in such circumstances ${ }^{12,41}$ (see Table 2). The mini-dose is most easily administered using an insulin syringe after reconstituting the glucagon with the diluent provided in the glucagon kit. The dose begins with $0.02 \mathrm{mg}$ (equal to $2 \mathrm{U}$ on an insulin syringe) for children up to age 2 years and then increases by $0.01 \mathrm{mg}$ ( $1 \mathrm{U}$ on an insulin syringe) per year of life up to a max dose of $0.15 \mathrm{mg}$ ( $15 \mathrm{U}$ on an insulin syringe). The mini-dose can be repeated after 30 to 60 minutes, if needed. If hypoglycemia persists and/or glucagon is not available, emergency services will be required for IV fluids containing dextrose. In the interim, simple sugar, like honey, molasses or cake frosting, can be given in the buccal cavity for rapid absorption by the oral mucosa, as long as there is no risk of aspiration. Of note, intranasal preparations of glucagon for easier administration have been studied and may become available soon. ${ }^{42}$

\section{5 | SPECIAL CONSIDERATIONS}

\section{1 | Insulin dose reductions for GI illnesses}

- If there is normoglycemia $(5-10 \mathrm{mmol} / \mathrm{L}$ or $90-180 \mathrm{mg} / \mathrm{dL})$ without ketone elevations, oral fluids with sugar should be offered along with appropriate insulin coverage, with ongoing monitoring of blood glucose and ketone levels every 2 hours. Frequent monitoring will guide insulin dosing reductions (see Table $1 \mathrm{~B}$ ).

- If there is normoglycemia $(5-10 \mathrm{mmol} / \mathrm{L}$ or $90-180 \mathrm{mg} / \mathrm{dL})$ with elevated blood or urine ketone levels, continue to give usual insulin doses with ongoing monitoring.

- If blood glucose is low ( $<3.9 \mathrm{mmol} / \mathrm{L}$ or $<70 \mathrm{mg} / \mathrm{dL}$ ), consider mini-dose glucagon.

- If vomiting persists beyond 2 hours, especially in children under 5 years old, or if hypoglycemia cannot be corrected, refer for IV fluids with dextrose along with continued monitoring.

\subsection{Specific advice regarding sick day management for children and adolescents receiving insulin pump therapy}

The key points of sick day management, mentioned previously, are the same for pump users as for those receiving insulin injections. ${ }^{20,43,44}$ Patients on pumps use only rapid- or short-acting insulin and do not have any injected depot of long-acting insulin so DKA can develop rapidly, with either interruption of insulin delivery, or during an intercurrent illness when no increased insulin is given. Episodes of hyperglycemia must be taken very seriously, especially if associated with elevated blood or urine ketones. If the blood glucose level is $14 \mathrm{mmol} / \mathrm{L}(\sim 250 \mathrm{mg} / \mathrm{dL})$ or above in a child or adolescent using an insulin pump, the following steps should be taken:

- Check for ketones in the blood or urine.

- Immediately check for problems with the pump or delivery system. Common problems include kinks in the catheter, air in the infusion line, disconnected catheters especially at the insertion site, and insertion site irritation. These can be identified when the patient and family change the infusion set immediately upon recognition of hyperglycemia that cannot otherwise be explained.

- Give a correction dose of insulin by syringe, based upon the blood glucose and ketone levels and not by the insulin pump, even after 
TABLE 3 Management of sick days and hyperglycemia with insulin pump ${ }^{8}[E]$

Ketones negative:
Give correction bolus with pump
Test BG hourly to confirm that BGs move downward
Drink low-carbohydrate fluids or salty liquids (ie, soup)
If BG lower after 1 hour, recheck again in 1-2 more hours
to decide if another bolus is needed
If BG not lower on recheck, then give bolus by syringe or
pen and follow instructions in second column

Blood ketones $>0.6 \mathrm{mmol} / \mathrm{L}$ or positive urine ketones or apparent that pump is not working or catheter blocked, dislodged etc:

May be a pump delivery or site problem or an illness.

Give sick day bolus by injection with pen or syringe using Table 1 guidelines for sick day booster $5-10-20 \%$ rule

Change the catheter, tubing, and insulin reservoir and check to be sure pump is working

Continue to follow sick day booster guidelines using pen or syringe until BGs respond

Re-establish insulin pump infusion with new set and cannula with temporary basal rate increase of approximately 120\%-150\% depending upon BG and ketone results

Monitor BG hourly and recheck ketones and weight at least every $4 \mathrm{~h}$

Drink extra high-carbohydrate fluids if the ketones are elevated and BG is low and extra low-carbohydrate "diet" fluids if BG is elevated with or without elevated ketones

If after $2 \mathrm{~h}$ there is no improvement, liaise with diabetes pump team. If after $2 \mathrm{~h}$ the BG is improved, use the unused bolus rule to decide if an additional bolus is needed ${ }^{\text {a }}$. Pump use can be resumed.

BG remains high, ketones persist, or nausea, vomiting, or abdominal pain develop, confusion or problems staying awake and alert, contact the diabetes pump team or proceed to immediate hospital assessment.

${ }^{a}$ Correction doses given for hyperglycemia should take into consideration the residual effect of any previous meal or correction bolus dose. A useful guide is to use the "unused bolus rule" (approximately 30\% of a rapid-acting insulin bolus is absorbed each hour). The correction dose should be reduced accordingly. For example, if 5 units had been given as a meal bolus $2 \mathrm{~h}$ previously, $60 \%$ would have been absorbed and the remaining $40 \%$ or 2 units would still be exerting an effect. This should be subtracted from any correction dose. However, most pumps have this "insulin on board" included in the "bolus guide," and you need then not subtract it manually if this function is activated.

the infusion set has been changed, in order to be certain that insulin is being delivered.

- Proceed with dosing and monitoring as directed in Table 3, depending on the glucose and ketone results. In cases of ketosis, always be sure to give the extra insulin with a pen or syringe to ensure delivery, and not with the pump, as malfunction may be the cause of ketosis.

- Subsequent correction boluses are increased by $10 \%$ to $20 \%$ during the period of illness, according to the glucose and ketone results, and can be given by pump once the infusion has been changed.

- To provide for extra basal insulin during sick days, the basal rate can be increased by $20 \%$ to $50 \%$, according to glucose and ketone results.

- Meal insulin boluses may need to be decreased during Gl illnesses, as noted above, when hypoglycemia is a concern.

- Basal insulin rates can also be decreased by $20 \%$ to $50 \%$ when hypoglycemia is a concern, as a temporary basal rate reduction for 2 to 4 hours or longer, as needed based on ongoing glucose and ketone monitoring.

\section{3 | Final considerations for sick day management in the current era of emerging technologies and adjunctive therapies}

There are many new advances for the care of young persons with diabetes, although regulatory approvals for the pediatric population often lag behind approvals for adults with diabetes. Nonetheless, the current era of diabetes management tools includes increasing use of CGM devices and emerging use of automated insulin delivery systems. Sick day management guidance should likely rely mainly on blood glucose results obtained by fingerstick along with blood ketone monitoring, preferably over urine ketone monitoring. However, certain CGM devices have received regulatory approval for nonadjunctive use; in other words, the CGM results can be used for management of low and high glucose levels. Due to the risk of DKA during sick days, it is wise to confirm CGM results with blood glucose monitoring to ensure timely delivery of extra insulin to avoid progression to DKA. However, CGM trend arrows may be helpful in managing sick days as the CGM device can signal whether the glucose is continuing to rise, fall, or is remaining stable. Recent guidelines have been published that recommend insulin dose adjustments according to the trend arrows on certain CGM devices. ${ }^{45}$ Children and adolescents using automated insulin delivery devices should likely change to manual mode during sick days in order to ensure adequate supplemental insulin delivery.

Finally, adjunctive use of the new class of oral agents called SGLT2 (or SGLT1/2) inhibitors have been reported to increase risk for DKA in patients with type 1 and type 2 diabetes. The greatest concern stems from the DKA risk, which can occur at times in the absence of extreme hyperglycemia, especially in the setting of "low carb" diets or low carbohydrate intake. ${ }^{46,47}$ Any patient receiving SGLT1/2 inhibitors must receive rigorous sick day management education to avoid progression to DKA. This includes training on the use of blood ketone monitoring of $\mathrm{BOHB}$ as atypical or euglycemic DKA has been reported and therefore typical blood glucose warning levels for DKA may be inadequate if SGLT1/2 inhibitors are being taken.

\section{Conflict of interest}

L.M.L. has consulting activities unrelated to the current manuscript with the following: AstraZeneca, Boehringer Ingelheim Pharmaceuticals, Inc., Dexcom, Inc., Eli Lilly and Company, Insulet, Johnson \& Johnson, MannKind Corporation, Merck, Novo Nordisk Inc., Roche 
Diagnostics. Sanofi U.S., and Unomedical. C.L. has consulting activities unrelated to the current manuscript with Sanofi and Eli Lilly. J.W. has research grants unrelated to the current manuscript from the following: AstraZeneca, Novo Nordisk, and Boehringer Ingelheim. S.E.H. has received lecturing honoraria from Eli Lilly and Sanofi. There are no reported conflicts from H.P. and A.V.

\section{ORCID}

Lori M. Laffel (D) http://orcid.org/0000-0002-9675-3001

Helen Phelan (D) http://orcid.org/0000-0002-4024-1912

Sabine E. Hofer (D) http://orcid.org/0000-0001-6778-0062

\section{REFERENCES}

1. Muller LM, Gorter KJ, Hak E, et al. Increased risk of infection in patients with diabetes mellitus type 1 or 2 . Ned Tijdschr Geneeskd. 2006;150:549-553.

2. Bagdade JD, Root RK, Bulger RJ. Impaired leukocyte function in patients with poorly controlled diabetes. Diabetes. 1974;23:9-15.

3. Liberatore RR, Barbosa SF, Alkimin MG, et al. Is immunity in diabetic patients influencing the susceptibility to infections? Immunoglobulins, complement and phagocytic function in children and adolescents with type 1 diabetes mellitus. Pediatr Diabetes. 2005;6:206-212.

4. Laffel L. Sick-day management in type 1 diabetes. Endocrinol Metab Clin North Am. 2000;29:707-723.

5. Cengiz E, Xing D, Wong JC, et al. Severe hypoglycemia and diabetic ketoacidosis among youth with type 1 diabetes in the T1D exchange clinic registry. Pediatr Diabetes. 2013;14(6):447-454.

6. Choudhary A. Sick day management in children and adolescents with type 1 diabetes. J Ark Med Soc. 2016;112(14):284-286.

7. Ilkowitz JT, Choi S, Rinke ML, Vandervoot K, Heptulla RA. Pediatric type 1 diabetes: reducing admission rates for diabetes ketoacidosis. Qual Manag Health Care. 2016;25(4):231-237.

8. Benoit SR, Zhang Y, Geiss LS, Gregg EW, Albright A. Trends in diabetic ketoacidosis hospitalizations and in-hospital mortality - United States, 2000-2014. MMWR Morb Mortal Wkly Rep. 2018;67(12):362-365.

9. Zargar AH, Wani Al, Masoodi SR, et al. Causes of mortality in diabetes mellitus: data from a tertiary teaching hospital in India. Postgrad Med J. 2009;85:227-232.

10. Walker M, Marshall SM, Alberti KG. Clinical aspects of diabetic ketoacidosis. Diabetes Metab Rev. 1989;5:651-663.

11. Soni A, Agwu JC, Wright NP, et al. Management of children with type 1 diabetes during illness: a national survey. Postgrad Med J. 2016; 92(1090):447-449.

12. Haymond MW, Schreiner B. Mini-dose glucagon rescue for hypoglycemia in children with type 1 diabetes. Diabetes Care. 2001;24:643-645.

13. Golden MP, Herrold AJ, Orr DP. An approach to prevention of recurrent diabetic ketoacidosis in the pediatric population. J Pediatr. 1985; 107:195-200.

14. Alexander $V$ on behalf of DiabNet, Scotland UK. Reducing DKA: a practical approach. J Pediatr Endocrinol Metab. 2002;15(suppl):22.

15. Farrell K, Holmes-Walker DJ. Mobile phone support is associated with reduced ketoacidosis in young adults. Diabetes Med. 2011;28:1001-1004.

16. Deeb A, Yousef H, Abdelrahman L, et al. Implementation of a diabetes educator care model to reduce paediatric admission for diabetic ketoacidosis. J Diabetes Res. 2016;2016:3917806.

17. Chiang JL, Kirkman MS, Laffel LM, Peters AL, Type 1 Diabetes Sourcebook Authors. Type 1 diabetes through the life span: a position statement of the American Diabetes Association. Diabetes Care. 2014; 37(7):2034-2054.

18. Brink SJ, Lee WR, Pillay, K, Kleinebreil, L. Diabetes in Children and Adolescents. Basic Training Manual for HealthCare Professionals in Developing Countries. Changing Diabetes in Children. NovoNordisk, Denmark, 2011.

19. Hanas R. Type 1 diabetes in children, adolescents and young adults. How to become an expert on your own diabetes. $3^{\text {rd }}$ ed. Class Publishing, London, England, 2007.
20. Chase HP, Maahs DM. Understanding diabetes. 13th ed. Denver, CO: Children's Diabetes Foundation; 2014.

21. Laffel L. Ketone bodies: a review of physiology, pathophysiology and application of monitoring to diabetes. Diabetes Metab Res Rev. 1999; 15:412-426.

22. Guerci B, Meyer L, Sallé A, et al. Comparison of metabolic deterioration between insulin analog and regular insulin after a 5-hour interruption of a continuous subcutaneous insulin infusion in type 1 diabetic patients. J Clin Endocrinol Metab. 1999;84(8):2673-2678.

23. Samuelsson U, Ludvigsson J. When should determination of ketonemia be recommended? Diabetes Technol Ther. 2002;4:645-650.

24. Guerci B, Tubiana-Rufi N, Bauduceau B, Bresson R, Cuperlier A, Delcroix C. Advantages to using capillary blood beta-hydroxybutyrate determination for the detection and treatment of diabetic ketosis. Diabetes Metab. 2005;31:401-406.

25. Guerci B, Benichou M, Floriot M, et al. Accuracy of an electrochemical sensor for measuring capillary blood ketones by fingerstick samples during metabolic deterioration after continuous subcutaneous insulin infusion interruption in type 1 diabetic patients. Diabetes Care. 2003; 26:1137-1141.

26. Umpierrez GE, Watts NB, Phillips LS. Clinical utility of betahydroxybutyrate determined by reflectance meter in the management of diabetic ketoacidosis. Diabetes Care. 1995;18:137-138.

27. Laffel L, Loughlin C, Tovar A, Zuehlke J, Brink S. Sick day management using blood beta hydroxybutyrate vs urine ketones significantly reduces hospital visits in youth with T1DM: a randomized clinical trial. Diabetes. 2002;51(suppl 2):A105.

28. Laffel LM, Wentzell K, Loughlin C, Tovar A, Moltz K, Brink S. Sick day management using blood 3-hydroxybutyrate (3-OHB) compared with urine ketone monitoring reduces hospital visits in young people with T1DM: a randomized clinical trial. Diabet Med. 2006;23:278-284.

29. Klocker AA, Phelan H, Twigg SM, Craig ME. Blood ß-hydroxybutyrate vs urine acetoacetate testing for the prevention and management of ketoacidosis in type 1 diabetes: a systematic review. Diabet Med. 2013;30:818-824.

30. Vanelli M, Chiari G, Capuano C, lovane B, Bernardini A, Giacalone T. The direct measurement of 3-beta-hydroxybutyrate enhances the management of diabetic ketoacidosis in children and reduces time and costs of treatment. Diabetes Nutr Metab. 2003;16:312-316.

31. Morris AD, Greene SA, Boyle DIR. Direct evidence of missing insulin as a cause of poor glycemic control and diabetic ketoacidosis. J Pediatr Endocrinol Metab. 1997;10:345-351.

32. Skinner TC. Recurrent diabetic ketoacidosis: causes, prevention and management. Horm Res. 2002;57(suppl 1):78-80.

33. Goebel-Fabbri AE. Diabetes and eating disorders. J Diabetes Sci Technol. 2008;2(3):530-532.

34. White K, Kolman ML, Wexler P. Unstable diabetes and unstable families: a psychosocial evaluation of diabetic children with recurrent ketoacidosis. Pediatrics. 1984;73:749-755.

35. Carter AW, Heinemann L. Insulin concentration in vials randomly purchased in pharmacies in the United States: considerable loss in the cold supply chain. J Diabetes Sci Technol. 2018;12(4):839-841.

36. Pouderoux $P$, Friedman N, Shirazi P, Ringelstein JG, Keshavarzian A. Effect of carbonated water on gastric emptying and intragastric meal distribution. Dig Dis Sci. 1997;42:34-39.

37. Basu A, Veettil S, Dyer R, Peyser T, Basu R. Direct evidence of acetaminophen interference with subcutaneous glucose sensing in humans: a pilot study. Diabetes Technol Ther. 2016;18(suppl 2): S2-43-S2-47.

38. Maahs DM, DeSalvo D, Pyle L, et al. Effect of acetaminophen on CGM glucose in an outpatient setting. Diabetes Care. 2015;38(10): e158-e159. https://doi.org/10.2337/dc15-1096.

39. Calhoun P, Johnson TK, Hughes J, Price D, Balo AK. Resistance to acetaminophen interference in a novel continuous glucose monitoring system. J Diabetes Sci Technol. 2018;12(2):393-396.

40. https://www.freestylelibre.us/cgm-reinvented. Accessed April 30, 2018.

41. Hartley M, Thomsett MJ, Cotterill AM. Mini-dose glucagon rescue for mild hypoglycaemia in children with type 1 diabetes: the Brisbane experience. J Paediatr Child Health. 2006;42:108-111. 
42. Sherr JL, Ruedy KJ, Foster NC, et al. Glucagon nasal powder: a promising alternative to intramuscular glucagon in youth with type 1 diabetes. Diabetes Care. 2016;39(4):555-562.

43. Walsh J, Roberts R. Pumping Insulin: Everything You Need for Success on a Smart Insulin Pump. 4th ed. San Diego, CA: Torrey Pines; 2006.

44. Kaufman FR. Insulin Pumps and Continuous Glucose Monitoring. 1st ed. Alexandria, VA: American Diabetes Association; 2012.

45. Laffel LM, Aleppo G, Buckingham BA, et al. A practical approach to using trend arrows on the Dexcom G5 CGM system to manage children and adolescents with diabetes. J Endocr Soc. 2017;1(12):1461-1476.

46. Peters AL, Buschur EO, Buse JB, Cohan P, Diner JC, Hirsch IB. Euglycemic diabetic ketoacidosis: a potential complication of treatment with sodium-glucose cotransporter 2 inhibition. Diabetes Care. 2015; 38(9):1687-1693.
47. Handelsman Y, Henry RR, Bloomgarden ZT, et al. American Association Of Clinical Endocrinologists and American College of Endocrinology position statement on the association of SGLT-2 inhibitors and diabetic ketoacidosis. Endocr Pract. 2016;22(6):753-762.

How to cite this article: Laffel LM, Limbert $C$, Phelan $H$, Virmani A, Wood J, Hofer SE. ISPAD Clinical Practice Consensus Guidelines 2018: Sick day management in children and adolescents with diabetes. Pediatr Diabetes. 2018;19 (Suppl. 27):193-204. https://doi.org/10.1111/pedi.12741 